\section{Genomic alterations in patients with somatic loss of the Y chromosome as the sole cytogenetic finding in bone marrow cells}

Madhu M. Ouseph,${ }^{1}$ Robert P. Hasserjian, ${ }^{2}$ Paola Dal Cin, ${ }^{1}$ Scott B. Lovitch, ${ }^{1}$ David P. Steensma, ${ }^{3}$ Valentina Nardi $^{2}$ and Olga K. Weinberg ${ }^{1,4, \infty}$

${ }^{1}$ Department of Pathology, Brigham and Women's Hospital; ' ${ }^{2}$ Pepartment of Pathology, Massachusetts General Hospital; ${ }^{3}$ Department of Medical Oncology, Dana-Farber Cancer Institute and 'Department of Pathology, Boston Children's Hospital, Boston, MA, USA

${ }^{\circ}$ Current address: Department of Pathology and Laboratory Medicine, Weill Cornell Medical College, New York, NY, USA

${ }^{\infty}$ Current address: UT Southwestern Medical Center, BioCenter, Dallas, TX, USA

\section{ABSTRACT}

L oss of the $\mathrm{Y}$ chromosome (LOY) is one of the most common somatic genomic alterations in hematopoietic cells in men. However, due to the high prevalence of LOY as the sole cytogenetic finding in the healthy older population, differentiating isolated LOY associated with clonal hematologic processes from aging-associated mosaicism can be difficult in the absence of definitive morphological features of disease. In the past, various investigators have proposed that a high percentage of metaphases with LOY is more likely to represent expansion of a clonal myeloid disease-associated population. It is unknown whether the proportion of metaphases with LOY is associated with the incidence of myeloid neoplasia-associated genomic alterations. To address this question, we identified bone marrow samples with LOY as an isolated cytogenetic finding and used targeted next generation sequencing-based molecular analysis to identify common myeloid neoplasia-associated somatic mutations. Among 73 patients with a median age of 75 years (range, 29-90), the percentage of metaphases with LOY was $<25 \%$ in 23 patients, $25-49 \%$ in $10,50-74 \%$ in 8 and $\geq 75 \%$ in 32 . A threshold of $\geq 75 \%$ LOY was significantly associated with a morphological diagnosis of myeloid neoplasm $(P=0.004)$. Furthermore, $\geq 75 \%$ LOY was associated with a higher lifetime incidence of a diagnosis of myelodysplastic syndromes (MDS) $(P<0.0001)$, and in multivariate analysis $\geq 75 \%$ LOY was a statistically significant independent predictor of myeloid neoplasia (odds ratio 6.17; 95\% confidence interval: 2.15-17.68; $P=0.0007]$. Higher LOY percentage $(\geq 75 \%)$ was associated with greater likelihood of having somatic mutations $(P=0.0009)$ and a higher number of these mutations $(P=0.0002)$. Our findings indicate that $\geq 75 \%$ LOY in bone marrow cells is associated with an increased likelihood of molecular aberrations in genes commonly seen to be altered in myeloid neoplasia and with morphological features of MDS. These observations suggest that $\geq 75 \%$ LOY in bone marrow should be considered an MDS-associated cytogenetic aberration.

\section{Introduction}

Loss of the Y chromosome (LOY) was first described in 1963 in cultured peripheral blood leukocytes. ${ }^{1} \mathrm{LOY}$ is one of the most common acquired somatic genomic alterations in men; present in approximately $6 \%$ of all male bone marrow karyotypes and in $16 \%$ of karyotypes from abnormal marrows. ${ }^{2,3}$ The incidence of LOY in bone marrow cells increases proportionally with age, with up to $20 \%$ of healthy men over 80 years of age showing LOY by conventional marrow karyotyping.,5

LOY is observed in association with myeloproliferative neoplasms (MPN), myelodysplastic syndromes (MDS), acute myeloid leukemias (AML) and B-cell
Ferrata Storti Foundation

Haematologica 2021

Volume 106(2):555-564

\section{Correspondence:}

OLGA K. WEINBERG

Olga.weinberg@UTSouthwestern.edu

Received: October 22, 2019.

Accepted: March 19, 2020.

Pre-published: March 19, 2020.

https://doi.org/10.3324/haematol.2019.240689

(C)2021 Ferrata Storti Foundation

Material published in Haematologica is covered by copyright. All rights are reserved to the Ferrata Storti Foundation. Use of published material is allowed under the following terms and conditions:

https://creativecommons.org/licenses/by-nc/4.0/legalcode. Copies of published material are allowed for personal or internal use. Sharing published material for non-commercial purposes is subject to the following conditions:

https://creativecommons. org//licenses/by-nc/4.0/legalcode, sect. 3. Reproducing and sharing published material for commercial purposes is not allowed without permission in writing from the publisher. 
lymphomas/leukemias, often in the context of other chromosomal abnormalities. ${ }^{2,6-8}$ LOY is one of the most common recurrent cytogenetic abnormalities seen in MDS, with an incidence of up to $30 \%$ in males. ${ }^{3,5,8-13}$ The incidence of isolated LOY in MDS is lower, ranging from 4 to $10 \%{ }^{8,14}$ Although there is no definitive association between LOY and clinical or biological characteristics in MDS, some groups have reported a higher incidence of ring sideroblasts, lower bone marrow myeloid to erythroid ratio, blast proportion, and blood leukocyte count in MDS patients with LOY than in those without LOY.9 MDS patients with isolated LOY have longer overall and leukemia-free survival compared to MDS patients with normal cytogenetics; conversely, in AML, chronic myeloid leukemia and chronic lymphocytic leukemia, isolated LOY has been associated with a poorer prognosis.,14-20 These observations suggest that MDS with LOY might be associated with a unique biology.

In individual cases, it can be difficult to distinguish whether LOY in MDS is a disease-associated alteration or just incidental aging-associated somatic mosaicism. In current World Health Organization (WHO) diagnostic criteria, LOY by itself cannot be used to define MDS, unlike other more strongly MDS-associated cytogenetic aberrations such as del(5q) or del $(7 q) .{ }^{21}$ Although the incidence of LOY increases with age, the percentage of metaphases with LOY in healthy people typically does not increase with age, so that a high proportion of metaphases may be more likely to represent a marker of disease rather than progressive clonal expansion of an inconsequential LOY cell population during aging. ${ }^{5,22}$ Some studies have suggested that $75-100 \%$ of metaphases with LOY in bone marrow are likely to be disease-associated rather than being age-associated. ${ }^{2,23,24}$

Recently, large genome-wide association studies enrolling more than 200,000 men in the UK Biobank demonstrated an association of germline single nucleotide polymorphisms of 156 autosomal loci as well as differential methylation of various genes with LOY., ${ }^{452-27}$ Whether any of the LOY-associated single nucleotide polymorphisms or epigenetic changes contribute to a risk of hematologic neoplasia is unclear.

While LOY involving a higher proportion of metaphases has been consistently associated with MDS, it is uncertain whether there is a corresponding increased incidence of pathogenic MDS-associated mutations, which typically affect genes involved in DNA methylation, DNA damage repair, chromatin modification, RNA processing, transcription and signal transduction. ${ }^{28}$ In addition, although karyotype evolution has been reported in patients with LOY over time, the rate at which patients with LOY accumulate additional mutations is unknown. ${ }^{23}$ The goal of this study was to evaluate the landscape of somatic mutations in patients with LOY.

\section{Methods}

The cytogenetic databases of Brigham and Women's Hospital and Massachusetts General Hospital (Boston, MA, USA) were searched for patients with isolated LOY cytogenetic abnormality on conventional karyotyping of bone marrow cells (defined as $\geq 3$ metaphases with isolated LOY) reported between 01/2005 and 08/2018. Patients with lymphoid or plasma cell malignancies involving bone marrow and those who had received chemother- apy within the preceding 3 months were excluded, as were those without a matching marrow specimen for morphological evaluation.

Medical records were reviewed to identify age at presentation, clinical diagnosis, and clinical course including treatment. Marrow aspirate smears and biopsy cores were re-evaluated by two hematopathologists (OW and $\mathrm{MO}$ ) and were classified according to the revised 2017 WHO classification of myeloid neoplasms ${ }^{21}$ Marrow samples that did not fulfill WHO-defined diagnostic criteria were separated into cases with minimal dysplasia (affecting $<10 \%$ cells in any lineage) and no dysplasia.

Fresh marrow aspirates at the time of initial presentation of LOY or concurrent blood samples were subjected to next-generation sequencing (NGS)-based molecular analysis for genes associated with hematologic neoplasms. For marrow samples that had not undergone NGS for clinical indications, NGS was performed on DNA extracted from the corresponding archived frozen cytogenetic cell pellets. NGS panels including 95 (Rapid Heme Panel of Brigham and Women's Hospital) or 103 (Heme SnapShot Panel of Massachusetts General Hospital) genes of importance in myeloid neoplasia (hotspots in oncogenes and most of the coding regions of tumor suppressors) (Online Supplementary Table S1) were used for mutation analysis. The former is an amplicon-based approach using an Illumina Truseq Custom Amplicon kit (San Diego, CA, USA), with an average depth of $1500 x .^{29}$ The latter is an anchored multiplex polymerase chain reaction-based panel (Archer DX, Boulder, CO, USA) with an average demultiplexed coverage of $350 \mathrm{x} .{ }^{30}$ After filtering for recurrent assay specific false positives, single nucleotide variants and small insertions/deletions at an allele fraction of $\geq 0.05$ were filtered based on population databases to eliminate likely germline variants. Somatic variants were further classified based on information available in literature, in silico tools, ClinVar (https://www.ncbi.nlm.nih.gov/clinvar), Catalog of Somatic Mutations in Cancer (https://cancer.sanger.ac.uk/cosmic) and published guidelines for the interpretation and reporting of sequence variants in cancer. ${ }^{31}$ Only likely pathogenic and pathogenic variants identified in patients' samples were analyzed for this study.

The selection of patients and analysis of clinical/laboratory results for this study were approved by the Partners Healthcare Institutional Review Board (Protocol \#: 2009P001369). Statistical analysis was performed using $\mathrm{JMP}^{\circledast}$, version 14 (SAS Institute Inc., Cary, NC, USA). A $P$-value of $<0.05$ was considered statistically significant. Individual $P$-values and tests performed are provided in the Results section. Briefly, in addition to descriptive statistics, analysis of distribution of categorical variables were performed using the Fisher exact test or Pearson $\chi^{2}$ test. Continuous variables were compared by one-way analysis of variance (ANOVA) or the Wilcoxon/Kruskal-Wallis test with $\chi^{2}$ approximation. A log-rank test was used to compare progression among patients with long-term follow-up. Multivariate analysis with a row-wise method for estimation of correlation and logistic regression analysis were used to compare predictor variables; and generate correlation coefficients, confidence intervals and correlation probabilities.

\section{Results}

\section{Identification of cases}

We identified 106 LOY cases reported as an isolated cytogenetic abnormality from 88 patients (Online Supplementary Figure S1A). Of these 106 samples, 15 were subsequently excluded from analysis because of lack of molecular analysis data (i.e., they failed the quality control 
for NGS assay). The final study set comprised the remaining 91 samples from 73 patients.

Age at presentation and extent of loss of chromosome $Y$ Most $(86 \%)$ patients were older than 65 years; the median age was 75 years (range, 29-90; mean 73 years) (Online Supplementary Figure S1B). Twenty-three patients had $<25 \%$ metaphases with LOY, 10 had 25-49\%, 8 had 50$74 \%$, and $32 \mathrm{had} \geq 75 \%$. Bivariate analysis did not demon- strate any significant correlation between age and percentage of metaphases involved by LOY (Table 1, Online Supplementary Figure S1C). All identified patients had undergone bone marrow evaluation for either a history of cytopenias or cytosis; 56 patients $(77 \%)$ had at least one cytopenia at the time of marrow evaluation. There was no association between percentage of metaphases with LOY and affected lineage or severity of peripheral blood cytopenias.

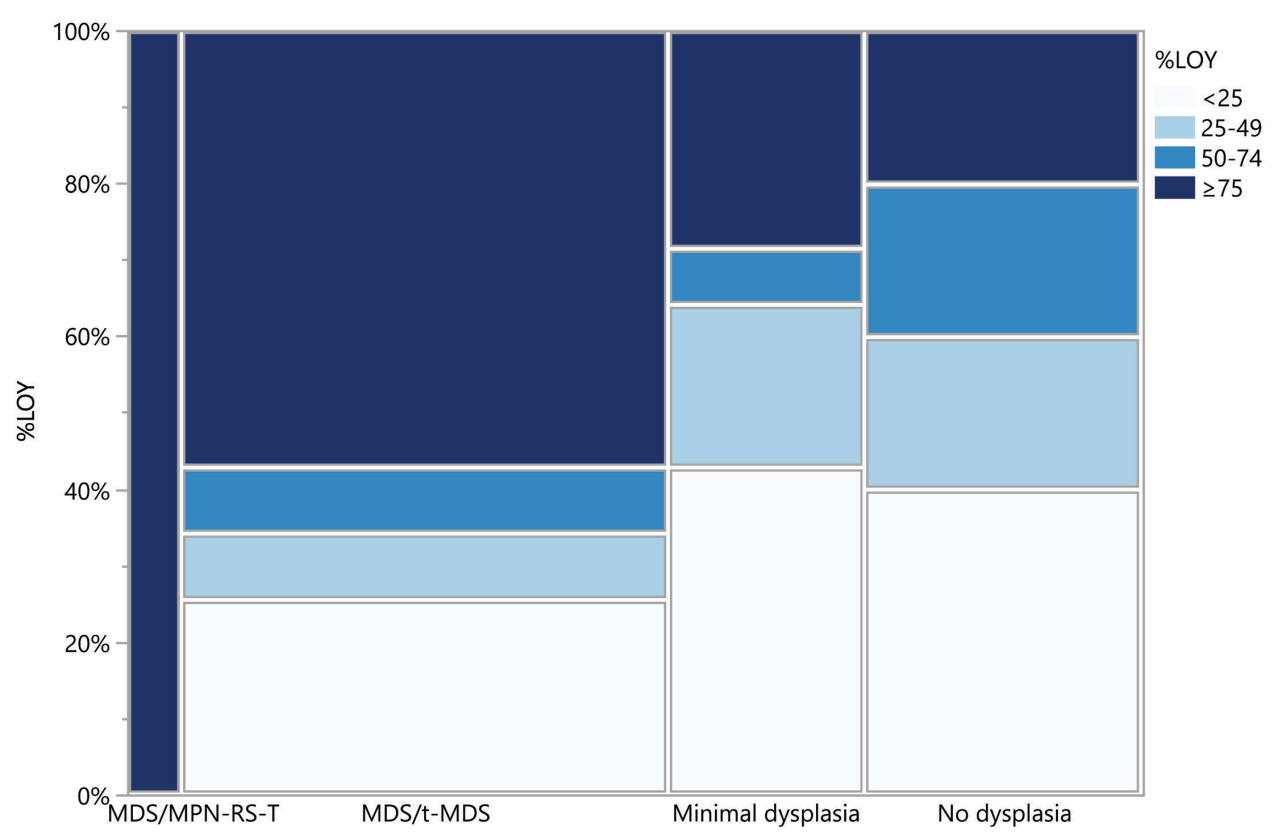

B

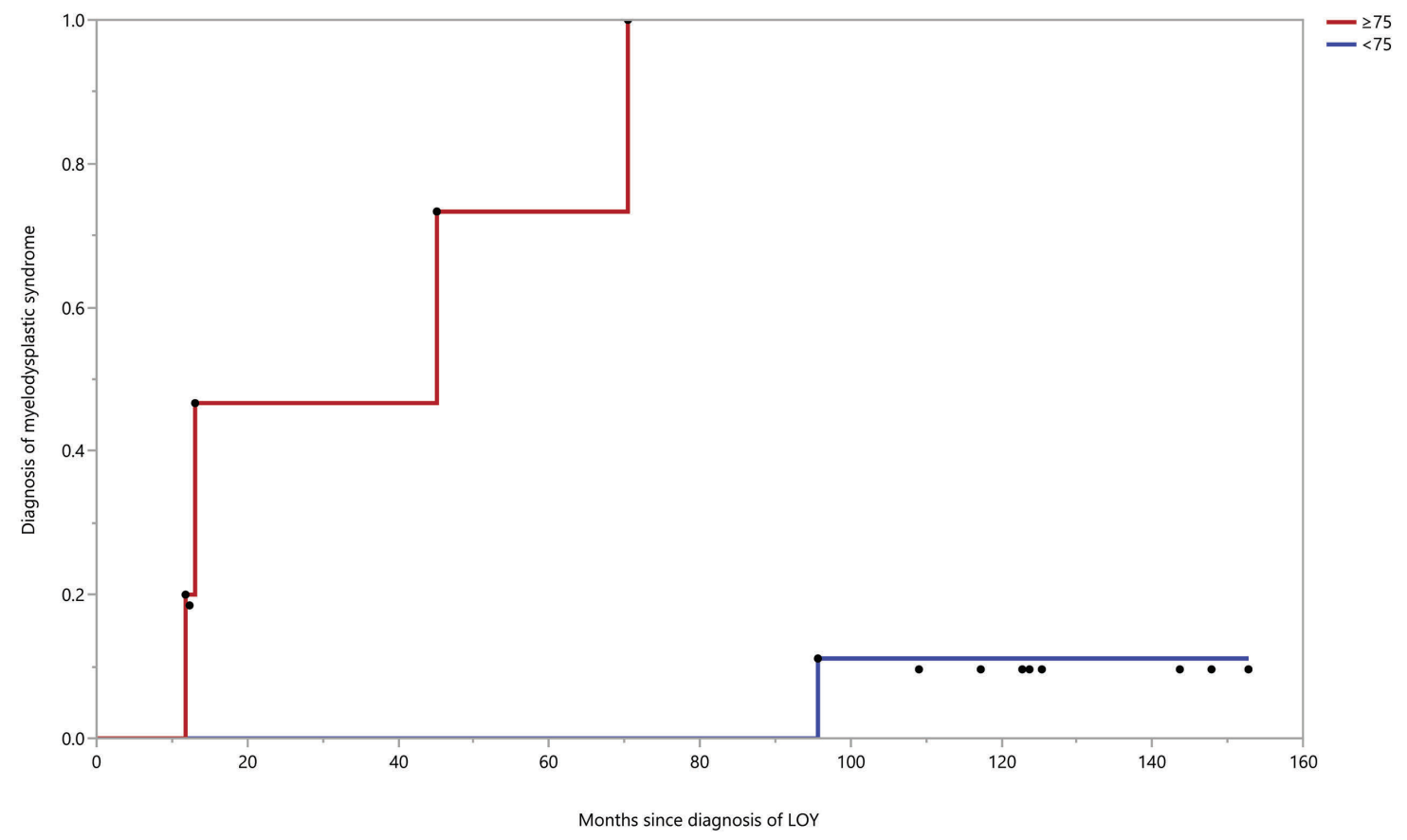

Figure 1. Loss of chromosome $Y$ in $\geq 75 \%$ metaphases is associated with morphological features of myeloid neoplasia and progression to myelodysplastic syndrome. (A) Mosaic graph demonstrating pathological diagnoses in relation to percentage of metaphases involved by loss of chromosome $Y$ (LOY) ; divided into four bins for ease of comparison. $\geq 75 \%$ LOY is significantly associated with myeloid neoplasia; while $<25 \%$ LOY is associated with no/minimal dysplasia. (B) Failure plot demonstrating presence or absence of evidence of progression in patients with LOY with no dysplasia/minimal dysplasia at presentation. Each dot represents diagnosis of myelodysplastic syndrome during follow up. The red line represents patients with $\geq 75 \%$ LOY, while the blue line represents patients with $<75 \%$ LOY. 
Loss of chromosome $Y$ in $\geq 75 \%$ metaphases is significantly associated with a morphological diagnosis of a myeloid neoplasm, especially myelodysplastic syndrome

A WHO-defined diagnosis was made in $39 / 73$ patients ( $53 \%$ of the total): 35 patients were diagnosed with different subtypes of MDS (MDS with single lineage dysplasia, MDS with multilineage dysplasia, MDS with ring sideroblasts and multilineage dysplasia, MDS with excess blasts1, MDS with excess blasts-2, and therapy-related MDS), as shown in Table 1. Four patients were diagnosed with myelodysplastic/myeloproliferative neoplasm with ring sideroblasts and thrombocytosis. No other MDS/MPN subtypes were identified and none of the patients met criteria for MPN or AML.

Among the remaining 34 patients who did not meet diagnostic criteria for any WHO-defined myeloid neoplasm, 14 had minimal dysplasia not meeting the 10\% diagnostic threshold for a diagnosis of MDS and one patient had a hypocellular bone marrow suggestive of aplastic anemia. The remaining 19 patients had a morphologically normal bone marrow evaluation. Of note, $73 \%$ of patients with minimal bone marrow dysplasia not diagnostic of MDS had blood cytopenias at presentation, while only $30 \%$ of patients with a morphologically normal bone marrow study had peripheral blood cytopenias; however, this difference did not reach statistical significance due to small numbers $(P=0.32$; Wilcoxon/KruskalWallis test, $\chi^{2}$ approximation).

There was a significant association between higher percentages of metaphases with LOY and diagnosis of myeloid neoplasia (Figure 1A). Bone marrow samples with $\geq 75 \%$ LOY had a very high likelihood of a morphological diagnosis of a myeloid neoplasm $(P=0.004$; Fisher exact test, Pearson $\chi^{2} P$-value), while samples with $<25 \%$ LOY were associated with no/minimal dysplasia ( $P=0.0484$; Fisher exact test, Pearson $\chi^{2} P$-value).

\section{Loss of chromosome $Y$ in $\geq 75 \%$ metaphases is significantly associated with progression to myelodysplastic syndrome}

Subsequent bone marrow evaluation had been performed on 34 patients who did not meet criteria for a WHO-defined diagnosis of myeloid neoplasia at the initial presentation with LOY, with an interval of 0.4 to 152.8 months (median 34.1 months) after the initial bone marrow evaluation. In the subsequent marrow evaluation, 4/7 patients $(57 \%$ ) with $\geq 75 \%$ LOY had a marrow morphological diagnosis of MDS, compared to only $1 / 27$ patients with $<75 \%$ LOY $\left(P<0.0001\right.$; log-rank test, $\left.\chi^{2}=22.979\right)$ (Figure 1B).

\section{Loss of chromosome $Y$ in $\geq 75 \%$ metaphases is significantly associated with mutations in myeloid neoplasia-related genes}

Among the 73 LOY patients with NGS performed at presentation, 25/32 (78\%) patients with $\geq 75 \%$ LOY had pathogenic mutations in tested myeloid neoplasia-associated genes. The frequency of mutations was associated with LOY burden: $5 / 8(63 \%)$ patients with $50-74 \%$ LOY, $5 / 10(50 \%)$ with $25-49 \%$ LOY, and $8 / 23$ (35\%) with $<25 \%$ LOY had mutations (Table 2, Online Supplementary Tables S2 and S3). The mean number of mutations per patient was 2.2 in samples with $\geq 75 \%$ LOY, 1.3 in samples with $50-74 \%$ LOY, 0.8 in samples with $25-49 \%$ LOY,
Table 1. Age and pathological diagnoses in the study population.

\begin{tabular}{lcccc} 
& \multicolumn{4}{c}{ [\%] LOY } \\
& $<25 \%$ & $25-49 \%$ & $50-74 \%$ & $\geq 75 \%$ \\
Age (years) & & & & \\
$25-49$ & 1 & 0 & 0 & 2 \\
$50-74$ & 11 & 4 & 4 & 11 \\
$75-100$ & 11 & 6 & 4 & 19 \\
Pathological diagnosis & & & & \\
No dysplasia & 8 & 4 & 4 & 4 \\
Minimal dysplasia, & 6 & 3 & 1 & 4 \\
not diagnostic of MDS & & & & \\
MDS/t-MDS & 9 & 3 & 3 & 20 \\
MDS-SLD & 6 & 1 & 1 & 4 \\
MDS-MLD & 2 & 2 & 1 & 10 \\
MDS-RS-MLD & 0 & 0 & 0 & 3 \\
MDS-EB-1 & 0 & 0 & 0 & 1 \\
$\quad$ MDS-EB-2 & 1 & 0 & 0 & 1 \\
t-MDS & 0 & 0 & 1 & 1 \\
MDS/MPN-RS-T & 0 & 0 & 0 & 4 \\
\hline
\end{tabular}

[\%] LOY: percentage of metaphases with loss of Y chromosome; MDS: myelodysplastic syndrome; MDS-SLD: MDS with single lineage dysplasia; MDS-MLD: MDS with multilineage dysplasia; MDS-RS-MLD: MDS with ring sideroblasts and multilineage dysplasia; MDS-EB-1: MDS with excess blasts-1; MDS-EB-2: MDS with excess blasts-2; t-MDS: therapy-related MDS; MDS/MPN-RS-T: myelodysplastic/myeloproliferative neoplasm with ring sideroblasts and thrombocytosis.

and 0.6 in samples with $<25 \%$ LOY. Bivariate analyses demonstrated that $\geq 75 \%$ LOY was significantly associated with greater likelihood of having somatic mutations commonly associated with myeloid neoplasm $(P=0.0009$; one-way ANOVA) and a higher number of such mutations $(P=0.0002$; one-way ANOVA) (Figure 2). Multivariate analysis demonstrated a positive correlation between total number of mutations as well as number of mutated genes and \% LOY (row-wise method) (Figure 3, Online Supplementary Tables S4 and S5). In multivariate analysis using a logistic regression model with effective likelihood ratio test, \% LOY was found to be a statistically significant predictor of diagnosis of myeloid neoplasia (odds ratio $[\mathrm{OR}] 1.03,95 \%$ confidence interval $[95 \% \mathrm{CI}]$ : 1.02-1.04; $P=0.0005)$ per $20 \%$ increase in LOY; OR 6.17 (95\% CI: $2.15-17.68 ; P=0.0007$ ) for $\geq 75 \%$ LOY) (Table 3 ).

The most commonly mutated genes were TET2, SF3B1, U2AF1, ZRSR2 and ASXL1 (Table 2, Online Supplementary Tables S2 and 3). Analysis of variance demonstrated that the variant allele frequencies (VAF) for pathogenic variants were significantly higher in patients with $\geq 75 \%$ LOY than in those with $<25 \%$ LOY $(P=0.000027$, F-ratio $=9.4$; one-way ANOVA) (Table 2). Patients with $\geq 75 \%$ LOY had an overall higher incidence $(50 \%)$ of mutations in spliceosome components (SFB31, SRSF2 and U2AF1), JAK2 and RUNX1 compared to the incidence of $9 \%$ in patients with LOY $<25 \%, 10 \%$ in those with $25-49 \%$ LOY and $13 \%$ in patients with $50-$ $74 \%$ LOY $\left(P=0.011\right.$; Pearson $\chi^{2}$ test) (Table 2). ${ }^{32}$ In addition, patients with $\geq 75 \%$ LOY had a higher incidence $(50 \%)$ of two or more mutations compared to the incidence of $9 \%$ of in patients with $<25 \%$ LOY, $10 \%$ in those with $25-49 \%$ LOY and $38 \%$ in patients with $50-74 \%$ LOY ( $P=0.01$; Pearson $\chi^{2}$ test) (Table 2). Furthermore, a large subset of patients with $\geq 75 \%$ LOY had these mutations in combination with mutations in TET2, DNMT3A and/or ASXL1 (28\%), compared to $4 \%$ of those with $<25 \%$ LOY, $10 \%$ of $25-49 \%$ LOY patients and $25 \%$ of $50-74 \%$ LOY patients $\left(P=0.01\right.$; Pearson $\chi^{2}$ test) (Table 2$)$. 
A

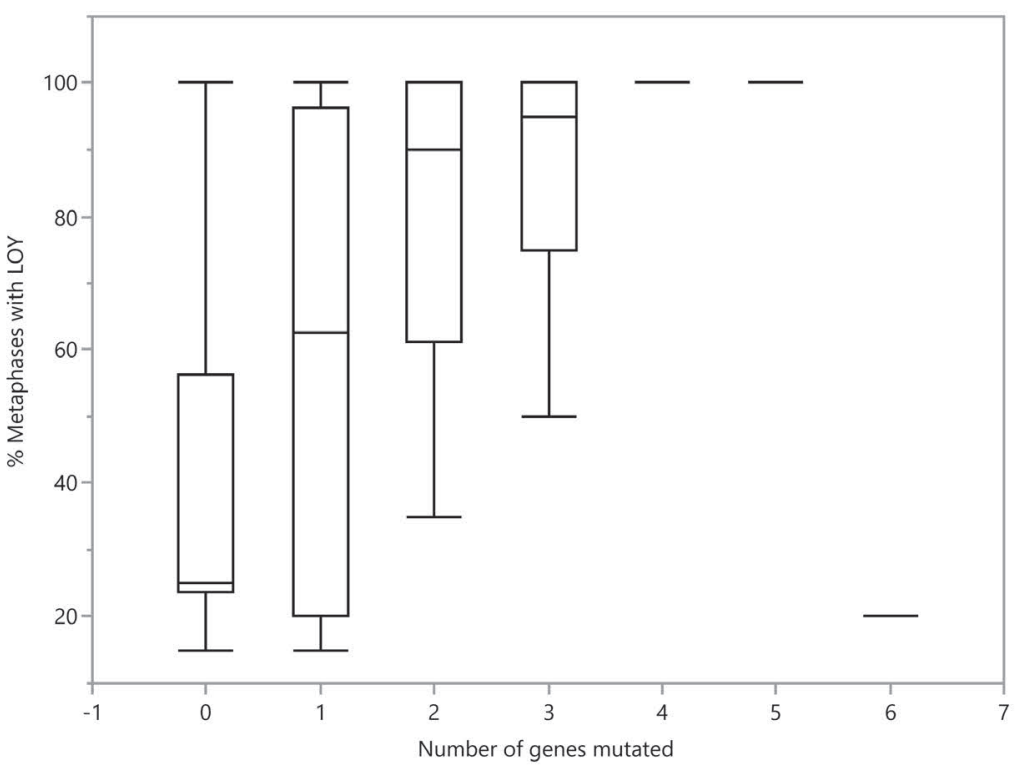

B

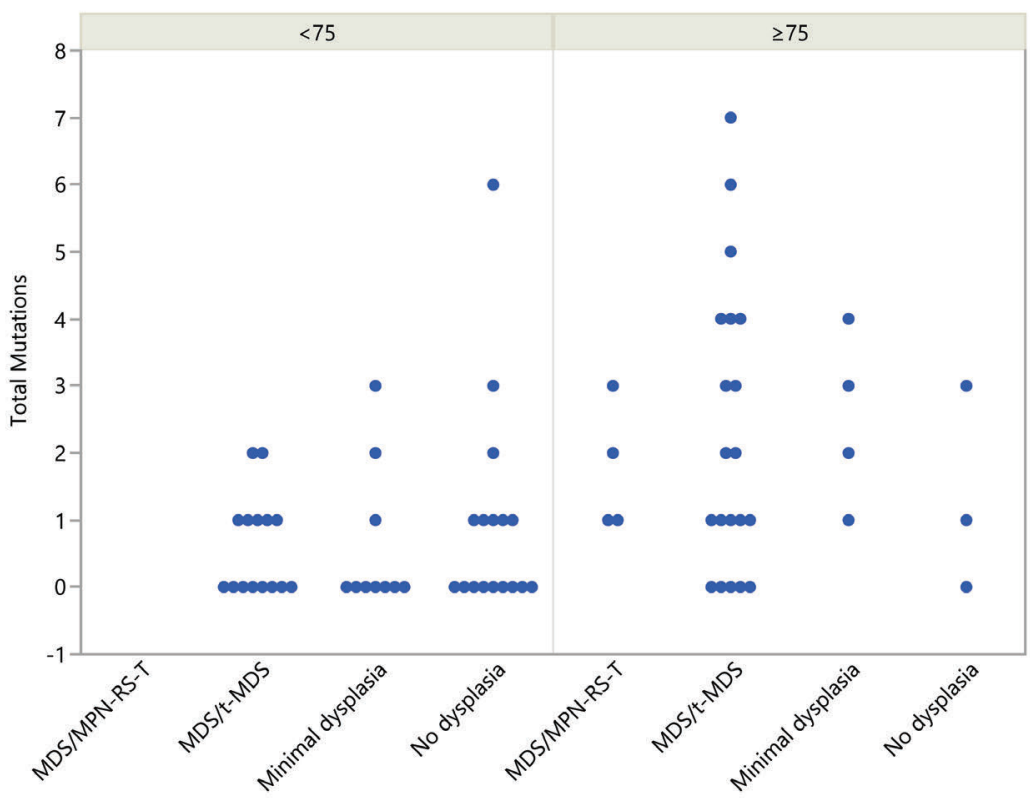

Figure 2. Loss of chromosome $Y$ in $\geq 75 \%$ metaphases is associated with somatic mutations in myeloid neoplasia-related genes. (A) Box plot demonstrating percentage of metaphases with loss of chromosome Y (LOY) compared to number of myeloid neoplasia related genes mutated. (B) Scatter plots demonstrating relation between total number of mutations and pathologic diagnosis in patients with $\geq 75 \%$ LOY and $<75 \%$. MDS/MPN-RS-T: myelodysplastic/myeloproliferative neoplasm with ring sideroblasts and thrombocytosis; MDS/t-MDS: myelodysplastic syndrome/therapy-related myelodysplastic syndrome.

Mutations with a high VAF as well as presence of two or more mutations, especially including mutations in TET2, $D N M T 3 A$ and/or ASXL1, are known to correlate strongly with the presence of MDS or to have a clinical course indistinguishable from that of MDS with the same mutation profile. ${ }^{32}$

Incidence of somatic mutations in patients with $\geq 75 \%$ loss of chromosome $Y$ is higher than that in the age-matched general population

We compared the proportion of patients with any mutation in myeloid neoplasia-related genes to the incidence of clonal hematopoiesis of indeterminate potential compiled from the published literature (Table 4). ${ }^{33}$ The overall incidence of mutations in myeloid neoplasia-related genes in our cohort of patients with any LOY was higher $(58 \%)$ than that seen in healthy individuals $>70$ years of age (10$15 \%)$. Moreover, the incidence was much higher when only patients with $\geq 75 \%$ LOY were considered $(81 \%)$, close to the incidence of somatic mutations seen in patients with MDS $(85-90 \%$ in most series with broad testing panels).

\section{Progression of loss of chromosome Y over time}

We identified 30 sequential samples with matching NGS data from 12 patients for evaluation of changes over time. These samples were obtained at varying time intervals after the first (median 22 months; range, 7-72 months). Of these 12 patients, seven did not demonstrate morphological features diagnostic of myeloid neoplasia at initial presentation with LOY (Figure 4). On follow-up, four of these seven patients progressed to having overt MDS, all of whom showed either an increased percentage of metaphases with LOY or persistent $100 \%$ LOY. The remaining three patients did not demonstrate evidence of progression to a myeloid neoplasm, despite an increase in the percentage of LOY metaphases in two. Of the five patients who had morphological diagnostic features of $\mathrm{MDS}$ at presentation, three maintained $100 \%$ LOY, while the remaining two showed an increase in percentage of LOY over the follow-up period. 


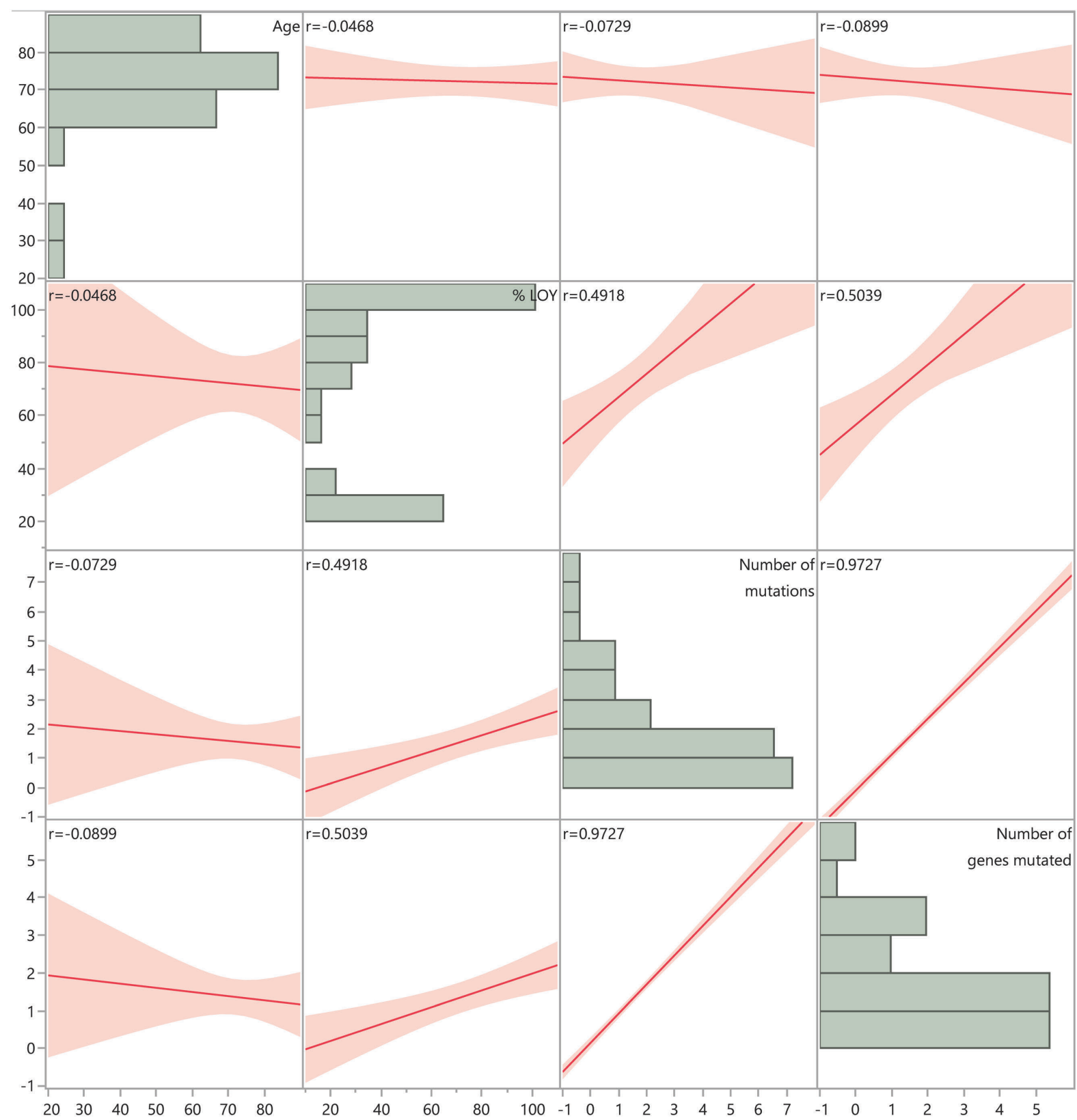

Figure 3. Percentage of loss of chromosome $\mathrm{Y}$ demonstrates positive correlation with total number of mutations as well as number of mutated genes by multivariate analysis. Scatterplot matrix demonstrating correlation between age, percentage of metaphases with loss of chromosome $\mathrm{Y}$, total number of mutations and number of genes mutated. Correlation coefficient for individual correlations provided in each scatterplot. Horizontal bar graphs demonstrating distribution of each parameter is also shown. LOY: loss of Y chromosome.

The number of somatic mutations identified by NGS was variable for patients over the follow-up period. Two out of three patients who did not demonstrate progression to MDS showed a loss of detectable mutations (with an initial KDM5A mutation with a VAF of 0.05 and $P P M 1 D$ with a VAF of 0.06 ) while the remaining patient showed no change in number of mutations. Of the four patients who showed progression to MDS, three had an increase in the number of mutations, while one patient did not demonstrate any change in number of mutations. Among five patients who had diagnostic morphological features of MDS at presentation, one patient showed a loss of detectable mutations (CUX1 with an initial VAF of 0.14 ) with persistence of diagnostic MDS morphological features, while one patient did not demonstrate any change in number of mutations over time. The remaining three patients showed increases in the numbers of mutations over the follow-up period. The mutations that accumulated over time in patients who had a diagnosis of MDS occurred in TET2 (2 patients, VAF 0.18 and 0.21), ZRSR2 (1 patient, VAF 0.14), RUNX1 (1 patient, VAF 0.05), ASXL1 (2 patients, VAF 0.36 and 0.09), SETBP1 (2 patients; VAF 0.24 and 0.18), STAG2 (1 patient; VAF 0.23), IDH1 (1 patient, VAF 0.31), STAT3 (1 patient, VAF 0.37), CBL (1 patient, VAF 0.23), CBLB (1 patient, VAF 0.13) and PHF6 (1 patient; VAF 0.29). 
Table 2. Loss of chromosome $Y$ is associated with somatic mutations that are frequently present in myeloid neoplasia.

\begin{tabular}{|c|c|c|c|c|}
\hline & \multicolumn{4}{|c|}{ [\%] LOY (total number of patients) } \\
\hline & $<25 \%(23)$ & $25-49 \%(10)$ & $50-74 \%(8)$ & $\geq 75 \%$ (32) \\
\hline \multicolumn{5}{|l|}{$\begin{array}{l}\text { Number of somatic mutations (number of patients with mutations; } \\
\text { mean VAF) }\end{array}$} \\
\hline TET2 & $2(1 ; 0.33)$ & 0 & $5(4 ; 0.36)$ & $12(9 ; 0.40)$ \\
\hline SF3B1 & $1(1 ; 0.23)$ & 0 & $1(1 ; 0.41)$ & $10(10 ; 0.36)$ \\
\hline U2AFI & 0 & 0 & 0 & $4(4 ; 0.25)$ \\
\hline ZRSR2 & 0 & 0 & 0 & $4(3 ; 0.48)$ \\
\hline ASXL1 & $1(1 ; 0.43)$ & $1(1 ; 0.05)$ & $1(1 ; 0.07)$ & $4(3 ; 0.32)$ \\
\hline$J A K 2$ & 0 & $1(1 ; 0.46)$ & 0 & $3(3 ; 0.37)$ \\
\hline SETBP1 & 0 & 0 & 0 & $2(2 ; 0.41)$ \\
\hline$C B L$ & 0 & 0 & $1(1 ; 0.49)$ & $2(2 ; 0.27)$ \\
\hline SH2B3 & 0 & 0 & 0 & $2(2 ; 0.27)$ \\
\hline STAG2 & 0 & 0 & 0 & $2(2 ; 0.31)$ \\
\hline Total number of somatic mutations (number of patients with mutations) & $13(7)$ & $7(5)$ & $9(5)$ & $65(25)$ \\
\hline $\begin{array}{l}\text { Number of patients with mutations in spliceosome components, } \\
\text { JAK2 and RUNXI (\%) }\end{array}$ & $2(8.7)$ & $1(10)$ & $1(12.5)$ & $16(50)$ \\
\hline Number of patients with $\geq 2$ mutations (\%) & $2(8.7)$ & $1(10)$ & $3(37.5)$ & $16(50)$ \\
\hline $\begin{array}{l}\text { Number of patients with combinations of mutations in TET2, } \\
\text { DNMT3A and/or ASXL1 (\%) }\end{array}$ & $1(4.4)$ & $1(10)$ & $2(25)$ & $9(28.1)$ \\
\hline
\end{tabular}

Number of mutations and variant allele fraction in commonly mutated myeloid neoplasia-related genes. For details please see Online Supplementary Tables S2 and S3. [\%] LOY: percentage of metaphases with loss of Y chromosome; VAF: variant allele fraction.

\section{Discussion}

LOY as a sole cytogenetic finding is one of the most common somatic genomic alterations in blood and marrow of elderly men., ${ }^{5,34}$ LOY as a post-zygote genomic alteration occurs at varying rates in different tissues, including non-hematopoietic cells. . $^{5,35,36}$ Interest in LOY is increasing because of the mounting evidence of a role of LOY in various disorders including atherosclerosis, latestage age-related macular degeneration, Alzheimer disease, and autoimmune disorders such as primary biliary cirrhosis and autoimmune thyroiditis. ${ }^{36-43}$ In addition, mosaic LOY in blood has been associated with a higher risk of all-cause mortality and overall shorter life expectancy. ${ }^{39,43-45}$ LOY in blood is associated with an increased incidence of concurrent diagnosis of nonhematopoietic malignancies such as head and neck, colorectal, prostatic and pancreatic carcinomas and may contribute to the increased incidence of some tumors in males. ${ }^{39,46-53}$ LOY in neoplastic tissues has been reported in various solid tumors, including carcinomas of urothelium, pancreas, esophagus, head and neck, and kidney. ${ }^{46,49,54-57}$

LOY is also one of the most common recurrent cytogenetic abnormalities seen in MDS. As MDS is predominantly observed in older people, it is difficult to separate age-associated LOY from disease-associated LOY. Although marrow sampling has an inherent age bias since the incidence of myeloid neoplasms increases with age, marrow samples taken from young patients evaluated for hematologic disorders do not demonstrate the same high incidence of LOY as that seen in the elderly. ${ }^{34}$ Consistent with published literature, in our cohort most patients were older than 65 years, with a median age of 75 years.

In an analysis conducted in 1992, in which conventional karyotyping was used, the incidence of LOY was estimated at approximately $11 \%$ in patients with MDS and $4 \%$ in those with AML. ${ }^{8}$ Subsequently, in 1997, GarciaIsidoro et al. demonstrated the presence of LOY in $29 \%$
Table 3. Multivariate analysis demonstrates that percentage loss of chromosome $Y$ is a statistically significant independent predictor of a diagnosis of myeloid neoplasia.

\begin{tabular}{|c|c|c|}
\hline Characteristic & Odds ratio (95\% CI) & $\begin{array}{c}\text { P-value } \\
\text { (effective likelihood } \\
\text { ratio test) }\end{array}$ \\
\hline$\%$ LOY & $\begin{array}{l}1.026(1.02-1.04) \text { per } 20 \% \text { increase } \\
8.83(2.51-34.89) \text { for entire range }\end{array}$ & 0.0005 \\
\hline$\geq 75 \%$ LOY & $6.17(2.15-17.68)$ & 0.0007 \\
\hline Age & $\begin{array}{l}0.99(0.95-1.03) \text { per } 10 \text { years } \\
0.60(0.04-7.87) \text { for entire range }\end{array}$ & 0.69 \\
\hline $\begin{array}{l}\text { Presence of } \\
\text { mutations }\end{array}$ & $2(0.78-5.15)$ & 0.15 \\
\hline $\begin{array}{l}\text { Number of } \\
\text { genes mutated }\end{array}$ & $\begin{array}{l}1.21(0.86-1.71) \text { per gene mutated } \\
3.12(0.40-24.53) \text { for entire range }\end{array}$ & 0.27 \\
\hline $\begin{array}{l}\text { Total number } \\
\text { of mutations }\end{array}$ & $\begin{array}{l}1.22(0.90-1.65) \text { per mutation } \\
4.02(0.48-33.35) \text { for entire range }\end{array}$ & 0.18 \\
\hline
\end{tabular}

Multivariate analysis using a logistic regression model for prediction of a diagnosis of myeloid neoplasia with odds of diagnosis of myeloid neoplasia, $95 \%$ confidence interval and $P$-value. 95\% CI: 95\% confidence interval; \% LOY: percentage of metaphases with loss of $Y$ chromosome).

of male patients diagnosed with MDS using fluorescence in situ hybridization studies on bone marrow. ${ }^{9}$

Some studies have suggested that the proportion of metaphases with LOY could be used to separate ageassociated LOY from disease-associated LOY. In previous analyses, the degree of LOY was not correlated with the severity of peripheral blood cytopenias. ${ }^{58,59}$ In an analysis published in 2000, Wiktor et al. identified that using $81 \%$ Y chromosome loss as a cut-off maximized the combined sensitivity (28\%) and specificity $(100 \%)$ for predicting the presence of disease states associated with LOY. ${ }^{2}$ In a subsequent study in 2011 by Wiktor and colleagues, restricted to men $>50$ years old, ${ }^{23}$ a myeloid neoplasm (MDS, MPN and AML) could be diagnosed in 64/161 patients with $>75 \%$ metaphases with LOY. ${ }^{23}$ In another series, a cutoff of $21.5 \%$ LOY in CD34-positive blood cells was proposed to discriminate between age- 
and disease-associated LOY in MDS, but CD34 selection for chromosome analysis is not used in the clinical setting. ${ }^{14}$

In our series, marrow samples with $\geq 75 \%$ LOY had a high likelihood of morphological diagnosis of myeloid neoplasia, most commonly MDS. Our data also suggest that patients with $\geq 75 \%$ LOY who initially have a nondiagnostic marrow have a higher likelihood of progression to a morphological diagnosis of MDS on follow-up. The presence of $<25 \%$ LOY metaphases, on the other hand, was associated with normal bone marrow morphology and a low likelihood of progression to MDS. These observations further support the notion that LOY involving a high proportion of metaphases might be a disease-associated genomic alteration.

To our knowledge, no published study has evaluated the presence of myeloid-type mutations in patients with isolated LOY. In our cohort, patients with $\geq 75 \%$ LOY had a high prevalence $(>80 \%)$ of somatic mutations associated with myeloid neoplasia, especially MDS. Although these mutations, as with LOY, can be seen in aging populations (so-called age-related clonal hematopoiesis or clonal hematopoiesis of indeterminate potential), the incidence of these frequent alterations was much higher when LOY was $\geq 75 \%$ compared to the incidence found in age-matched populations from the literature. These findings suggest that $\geq 75 \%$ LOY is associated with an MDS-type mutation signature and therefore likely represents disease-associated clonal proliferation. ${ }^{22}$

The molecular mechanisms behind the association of LOY, aging and neoplasia are not completely understood. There is no clear association between LOY and numerical abnormalities of other chromosomes. ${ }^{9,27,53}$ LOY does not seem to reflect an overall propensity to loss of small-sized chromosomes. ${ }^{60,61}$ Alternative proposed causes for a high
Table 4. Prevalence of mutations in myeloid neoplasia-related genes in the cohort with $\geq 75 \%$ loss of chromosome $Y$ is comparable to the reported prevalence in patients with myelodysplastic syndrome.

\begin{tabular}{|c|c|c|c|c|}
\hline & \multicolumn{4}{|c|}{ Mutation frequency } \\
\hline & $\begin{array}{l}\text { Myelodysplastic } \\
\text { syndrome }\end{array}$ & $\begin{array}{l}\text { Current } \\
\text { LOY cohort }\end{array}$ & $\begin{array}{l}\text { Current } \geq 75 \% \\
\text { LOY cohort }\end{array}$ & $\begin{array}{l}\text { Healthy } \\
\text { individuals } \\
>70 \text { years }\end{array}$ \\
\hline $\begin{array}{l}\text { Proportion of patients } \\
\text { with any mutation }\end{array}$ & $72-90 \%$ & $58 \%$ & $81 \%$ & $5-10 \%$ \\
\hline $\begin{array}{c}\text { Splicing factor genes } \\
\text { SF3B1 } \\
\text { SRSF2 } \\
\text { U2AF1 } \\
\text { ZRSR2 }\end{array}$ & $\begin{array}{l}15-32 \% \\
10-17 \% \\
7-12 \% \\
3-11 \%\end{array}$ & $\begin{array}{l}12 \% \\
2 \% \\
4 \% \\
4 \% \\
\end{array}$ & $\begin{array}{c}32 \% \\
3 \% \\
13 \% \\
10 \% \\
\end{array}$ & $\begin{array}{l}0.1-0.2 \% \\
<0.1 \% \\
<0.1 \%\end{array}$ \\
\hline $\begin{array}{l}\text { DNA methylation gen } \\
\text { TET2 } \\
\text { DNMT3A }\end{array}$ & $\begin{array}{l}20-32 \% \\
5-13 \%\end{array}$ & $\begin{array}{l}13 \% \\
4 \%\end{array}$ & $\begin{array}{l}26 \% \\
3 \%\end{array}$ & $\begin{array}{c}0.3-0.4 \% \\
1.5-2 \%\end{array}$ \\
\hline $\begin{array}{l}\text { Histone modification } \\
\text { ASXL1 } \\
\text { EZH2 }\end{array}$ & $\begin{array}{l}\text { genes } \\
\qquad \begin{array}{c}11-23 \% \\
5-12 \%\end{array}\end{array}$ & $\begin{array}{l}6 \% \\
2 \%\end{array}$ & $\begin{array}{l}10 \% \\
7 \%\end{array}$ & $0.3-0.4 \%$ \\
\hline $\begin{array}{l}\text { Transcription factors } \\
\text { SETBP1 } \\
\text { BCOR/BCORL }\end{array}$ & $\begin{array}{c}2-5 \% \\
4 \%\end{array}$ & $\begin{array}{l}2 \% \\
3 \%\end{array}$ & $\begin{array}{l}7 \% \\
3 \%\end{array}$ & $<0.1 \%$ \\
\hline $\begin{array}{l}\text { Cohesin complex ger } \\
\text { STAG1/STAG2 }\end{array}$ & $4-7 \%$ & $1 \%$ & $3 \%$ & \\
\hline $\begin{array}{l}\text { Signaling genes } \\
\quad N R A S / K R A S \\
C B L \\
\text { JAK2 }\end{array}$ & $\begin{array}{l}5-10 \% \\
2-5 \% \\
2-5 \%\end{array}$ & $\begin{array}{l}2 \% \\
3 \% \\
4 \%\end{array}$ & $\begin{array}{l}3 \% \\
7 \% \\
7 \%\end{array}$ & $\begin{array}{c}<0.1 \% \\
0.2 \%\end{array}$ \\
\hline $\begin{array}{l}\text { Tumor suppressor ge } \\
\text { TP53 }\end{array}$ & $5-10 \%$ & $2 \%$ & $3 \%$ & $<0.1 \%$ \\
\hline
\end{tabular}

Comparison of mutation frequency in patients with loss of chromosome $Y$ with a reported incidence of mutations in the healthy aging population and patients with myelodysplastic syndrome.The table is adapted from Table $45-3$ of the paper by Hasserjian et al..$^{33} \%$ LOY: percentage of metaphases with loss of Y chromosome.

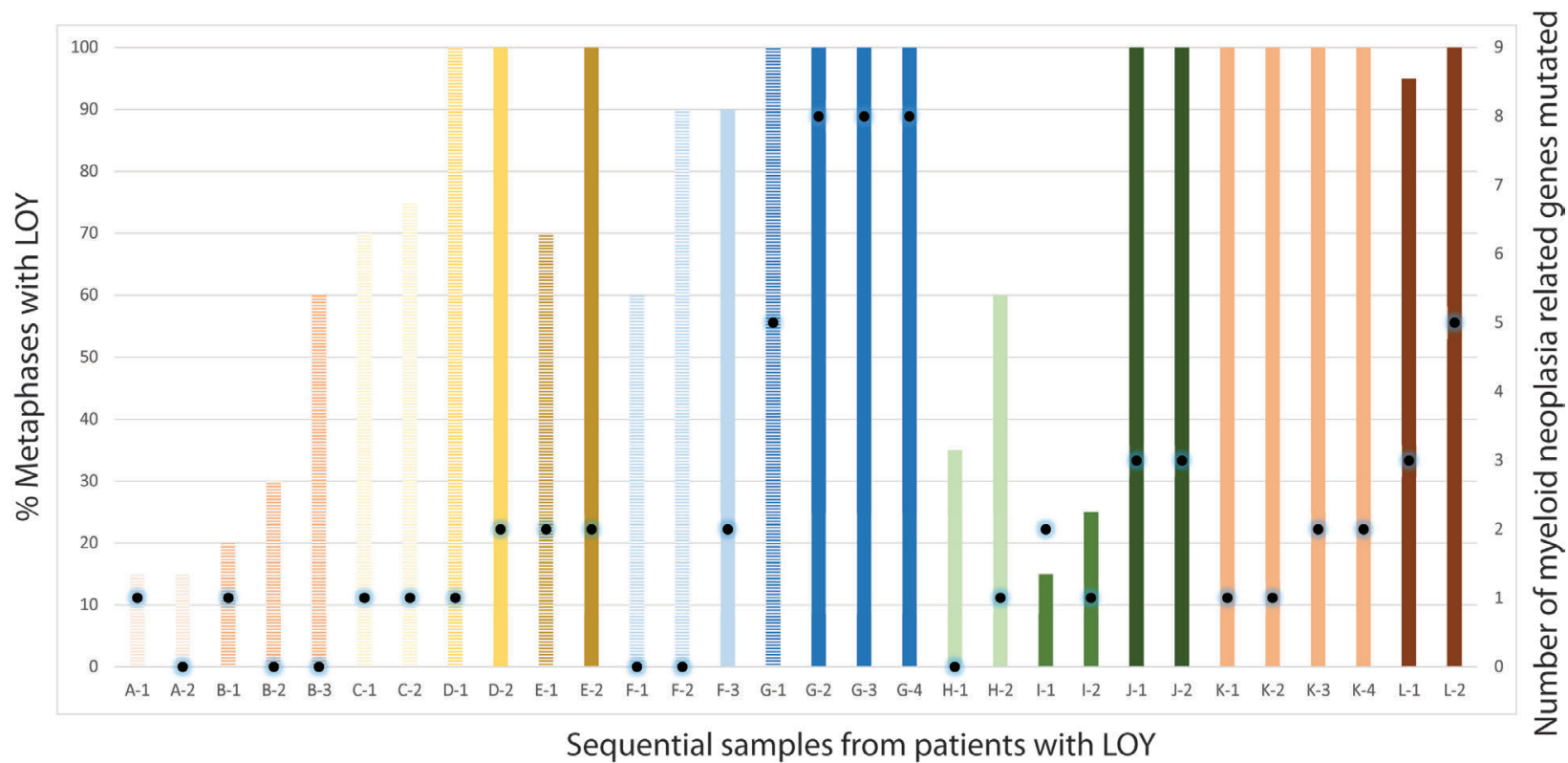

Figure 4. Progression of somatic mutations in 12 patients with loss of chromosome Y. Striped bars represent a pathological diagnosis of no dysplasia or minimal dysplasia, while solid bars represent pathological diagnosis of myelodysplastic syndrome (Each alphabet letter corresponds to one patient). The height of bars represents the percentage of metaphases with loss of chromosome Y. Black dots on bars highlight number of mutations identified for the corresponding samples. LOY: loss of $Y$ chromosome. 
incidence of LOY with aging include the presence of shortened telomeres as well as late S-phase replication of the Y chromosome. ${ }^{38,62}$ Smoking appears to be the most common epidemiological association with mosaic LOY in the bone marrow. ${ }^{4,25,63}$

It is unclear how LOY contributes to the phenotypes associated with LOY. LOY may not be limited to myeloid lineages, as more frequent LOY has been reported in CD3-positive cells in the blood of elderly patients with MDS compared to those without MDS. ${ }^{14}$ While some investigators have suggested an immune regulatory component, deregulation of proteins encoded by the male-specific region of the $\mathrm{Y}$ chromosome region has been seen in neoplasms with LOY, raising the possibility of a direct function of these proteins in carcinogenesis. ${ }^{48,64,65}$ In vitro models have demonstrated a tumor suppressor role of the Y chromosome in the context of prostatic carcinoma. ${ }^{66}$ Furthermore, identification of tumor suppressor roles of ZFY and UTY, which localize to the male-specific part of $Y$, suggests a more active role of LOY in oncogenesis. ${ }^{53}$ Both these genes have homologues on the $\mathrm{X}$ chromosome which escape $\mathrm{X}$ inactiva- tion. ${ }^{53,67}$ However, constitutional 45, XO (Turner syndrome) is not associated with an increased incidence of myeloid neoplasms. ${ }^{68}$

In conclusion, our findings demonstrate that a high proportion of metaphases with LOY ( $\geq 75 \%$ metaphases) in marrow is associated with a high frequency of molecular alterations in genes commonly mutated in myeloid neoplasia and strongly associated with a contemporaneous or subsequent diagnosis of MDS. These observations suggest that high-proportion LOY is likely to be a true MDSassociated cytogenetic aberration rather than an incidental finding due to aging.

\section{Disclosures}

No conflicts of interest to diclose.

\section{Contributions}

MO collected cases and compiled data. MO and OW reviewed cases. OW, RH and VN contributed cases. VN and SL analyzed NGS data. PC oversaw cytogenetic testing. DS provided clinical information related to cases. All authors contributed to the preparation of the manuscript and the revision.

\section{References}

1. Jacobs PA, Brunton M, Court Brown WM, Doll R, Goldstein H. Change of human chromosome count distribution with age: evidence for a sex differences. Nature. 1963;197:1080-1081.

2. Wiktor A, Rybicki BA, Piao ZS, et al. Clinical significance of $Y$ chromosome loss in hematologic disease. Genes Chromosomes Cancer. 2000;27(1):11-16

3. Shahrabi S, Khodadi E, Saba F, Shahiahani M, Saki N. Sex chromosome changes in leukemia: cytogenetics and molecular aspects. Hematology. 2018;23(8):139-147.

4. Wright DJ, Day FR, Kerrison ND, et al. Genetic variants associated with mosaic $Y$ chromosome loss highlight cell cycle genes and overlap with cancer susceptibility. Nat Genet. 2017;49(5):674-679

5. Pierre RV, Hoagland HC. Age-associated aneuploidy: loss of $\mathrm{Y}$ chromosome from human bone marrow cells with aging. Cancer. 1972:30(4):889-894.

6. Zhang LJ, Shin ES, Yu ZX, Li SB. Molecular genetic evidence of $Y$ chromosome loss in male patients with hematological disorders. Chin Med J (Engl). 2007;120(22):2002-2005.

7. Abe S, Golomb HM, Rowley JD, Mitelman F, Sandberg AA. Chromosomes and causation of human cancer and leukemia. XXXV. The missing $\mathrm{Y}$ in acute non-lymphocytic leukemia (ANLL). Cancer. 1980;45(1):84-90.

8. Loss of the Y chromosome from normal and neoplastic bone marrows. United Kingdom Cancer Cytogenetics Group (UKCCG). Genes Chromosomes Cancer. 1992;5(1):8388.

9. Garcia-Isidoro $M$, Tabernero $\mathrm{MD}$, Najera $\mathrm{ML}$, et al. Clinical and biological characteristics of myelodysplastic syndromes with nulisomy Y by FISH. Haematologica. 1997;82(5):537-541.

10. Haase D, Fonatsch C, Freund $M$, et al. Cytogenetic findings in 179 patients with myelodysplastic syndromes. Ann Hematol. 1995;70(4):171-87.

11. Levan G, Mitelman F. Clustering of aberra- tions to specific chromosomes in human neoplasms. Hereditas. 1975;79(1):156-160.

12. Suciu S, Kuse R, Weh HI, Hossfeld DK. Results of chromosome studies and their relation to morphology, course, and prognosis in 120 patients with de novo myelodysplastic syndrome. Cancer Genet Cytogenet. 1990;44(1):15-26

13. Nomdedeu M, Pereira A, Calvo X, et al. Clinical and biological significance of isolated Y chromosome loss in myelodysplastic syndromes and chronic myelomonocytic leukemia. A report from the Spanish MDS Group. Leuk Res. 2017;63:85-89.

14. Ganster C, Kampfe D, Jung K, et al. New data shed light on Y-loss-related pathogenesis in myelodysplastic syndromes. Genes Chromosomes Cancer. 2015;54(12):717724.

15. Lippert E, Etienne G, Mozziconacci MJ, et al. Loss of the Y chromosome in Philadelphiapositive cells predicts a poor response of chronic myeloid leukemia patients to imatinib mesylate therapy. Haematologica. 2010;95(9):1604-1607.

16. Holmes RI, Keating MJ, Cork A, Trujillo JM, McCredie KB, Freireich EJ. Loss of the Y chromosome in acute myelogenous leukemia: a report of 13 patients. Cancer Genet Cytogenet. 1985;17(3):269-278.

17. Chapiro E, Antony-Debre I, Marchay N, et al. Sex chromosome loss may represent a disease-associated clonal population in chronic lymphocytic leukemia. Genes Chromosomes Cancer. 2014;53(3):240-247.

18. Greenberg P, Cox C, LeBeau MM, et al. International scoring system for evaluating prognosis in myelodysplastic syndromes. Blood. 1997:89(6):2079-2088.

19. Slovak ML, Kopecky KJ, Cassileth PA, et al. Karyotypic analysis predicts outcome of preremission and postremission therapy in adult acute myeloid leukemia: a Southwest Oncology Group/Eastern Cooperative Oncology Group Study. Blood. 2000;96(13): 4075-4083.

20. Schanz J, Tüchler H, Solé F, et al. New comprehensive cytogenetic scoring system for primary myelodysplastic syndromes (MDS) and oligoblastic acute myeloid leukemia after MDS derived from an international database merge. J Clin Oncol. 2012;30(8): 820-829.

21. Swerdlow SH. WHO Classification of Tumours of Haematopoietic and Lymphoid Tissues. International Agency for Research on Cancer. 2017.

22. Danielsson M, Halvardson J, Davies H, et al. Longitudinal changes in the frequency of mosaic chromosome $Y$ loss in peripheral blood cells of aging men varies profoundly between individuals. Eur J Hum Genet. 2020;28(3):349-357.

23. Wiktor AE, Van Dyke DL, Hodnefield JM, Eckel-Passow J, Hanson CA. The significance of isolated $\mathrm{Y}$ chromosome loss in bone marrow metaphase cells from males over age 50 years. Leuk Res. 2011;35(10):1297-300.

24. Wong AK, Fang B, Zhang L, Guo X, Lee S, Schreck R. Loss of the Y chromosome: an age-related or clonal phenomenon in acute myelogenous leukemia/myelodysplastic syndrome? Arch Pathol Lab Med. 2008;132(8):1329-1332.

25. Zhou W, Machiela MJ, Freedman ND, et al Mosaic loss of chromosome $\mathrm{Y}$ is associated with common variation near TCL1A. Nat Genet. 2016;48(5):563-568

26. Terao C, Momozawa Y, Ishigaki $\mathrm{K}$, et al GWAS of mosaic loss of chromosome $Y$ highlights genetic effects on blood cell differentiation. Nat Commun. 2019:10(1):4719.

27. Thompson DJ, Genovese G, Halvardson J, et al. Genetic predisposition to mosaic Y chromosome loss in blood. Nature. 2019;575(7784):652-657.

28. Ogawa S. Genetics of MDS. Blood. 2019;133(10):1049-1059.

29. Kluk MJ, Lindsley RC, Aster JC, et al. Validation and implementation of a custom next-generation sequencing clinical assay for hematologic malignancies. J Mol Diagn. 2016;18(4):507-515

30. Zheng Z, Liebers M, Zhelyazkova B, et al. Anchored multiplex PCR for targeted nextgeneration sequencing. Nat Med. 2014:20(12):1479-1484 
31. Li MM, Datto M, Duncavage EJ, et al. Standards and guidelines for the interpretation and reporting of sequence variants in cancer: a joint consensus recommendation of the Association for Molecular Pathology, American Society of Clinical Oncology, and College of American Pathologists. J Mol Diagn. 2017;19(1):4-23.

32. Malcovati L, Galli A, Travaglino E, et al. Clinical significance of somatic mutation in unexplained blood cytopenia. Blood. 2017;129(25):3371-3378.

33. Hasseriian RP, Head DR. Myelodysplastic syndromes. In: Jaffe EA, Arber DA; Campo E; Harris, NL; Quintanilla-Martinez, L, editors Hematopathology. Second edition Elsevier, Inc. 2017:793-815.

34. Nowinski GP, Van Dyke DL, Tilley BC, et al. The frequency of aneuploidy in cultured lymphocytes is correlated with age and gender but not with reproductive history. Am J Hum Genet. 1990:46(6):1101-1111.

35. Forsberg LA, Halvardson J, RychlickaBuniowska E, et al. Mosaic loss of chromosome $\mathrm{Y}$ in leukocytes matters. Nat Genet. 2019;51(1):4-7.

36. Haitjema S, Kofink D, van Setten J, et al. Loss of $Y$ chromosome in blood is associated with major cardiovascular events during follow-up in men after carotid endarterectomy. Circ Cardiovasc Genet. 2017;10(4): e001544.

37. Lleo A, Oertelt-Prigione S, Bianchi I, et al. Y chromosome loss in male patients with primary biliary cirrhosis. J Autoimmun. 2013;41:87-91.

38. Persani L, Bonomi M, Lleo A, et al. Increased loss of the $\mathrm{Y}$ chromosome in peripheral blood cells in male patients with autoimmune thyroiditis. J Autoimmun. 2012;38(23):J193-196.

39. Forsberg LA, Rasi C, Malmqvist N, et al. Mosaic loss of chromosome $Y$ in peripheral blood is associated with shorter survival and higher risk of cancer. Nat Genet. 2014;46(6):624-628.

40. Charchar FJ, Bloomer LD, Barnes TA, et al. Inheritance of coronary artery disease in men: an analysis of the role of the Y chromosome. Lancet. 2012;379(9819):915-922.

41. Grassmann F, Kiel C, den Hollander AI, et al. $\mathrm{Y}$ chromosome mosaicism is associated with age-related macular degeneration. Eur J Hum Genet. 2019;27(1):36-41

42. Grassmann F, International AMDGC, Weber BHF, Veitia RA. Insights into the loss of the $Y$ chromosome with age in control individuals and in patients with age-related macular degeneration using genotyping microarray data. Hum Genet. 2020;139(3):401-407.

43. Dumanski JP, Lambert JC, Rasi C, et al. Mosaic loss of chromosome $\mathrm{Y}$ in blood is associated with Alzheimer disease. Am J Hum Genet. 2016;98(6):1208-1219.

44. Forsberg LA. Loss of chromosome Y (LOY) in blood cells is associated with increased risk for disease and mortality in aging men. Hum Genet. 2017;136(5):657-663.

45. Loftfield E, Zhou W, Graubard BI, et al Predictors of mosaic chromosome $Y$ loss and associations with mortality in the UK Biobank. Sci Rep. 2018;8(1):12316.

46. Veiga LC, Bergamo NA, Reis PP, Kowalski LP, Rogatto SR. Loss of Y-chromosome does not correlate with age at onset of head and neck carcinoma: a case-control study. Braz J Med Biol Res. 2012:45(2):172-178.

47. Noveski P, Madjunkova S, Sukarova Stefanovska E, et al. Loss of Y chromosome in peripheral blood of colorectal and prostate cancer patients. PLoS One. 2016;11(1):e0146264.

48. Kido T, Lau YF. Roles of the Y chromosome genes in human cancers. Asian J Androl. 2015;17(3):373-380.

49. Hunter S, Gramlich T, Abbott K, Varma V. Y chromosome loss in esophageal carcinoma: an in situ hybridization study. Genes Chromosomes Cancer. 1993:8(3):172-177.

50. Park SJ, Jeong SY, Kim HJ. Y chromosome loss and other genomic alterations in hepatocellular carcinoma cell lines analyzed by $\mathrm{CGH}$ and $\mathrm{CGH}$ array. Cancer Genet Cytogenet. 2006;166(1):56-64.

51. Cook MB, McGlynn KA, Devesa SS, Freedman ND, Anderson WF. Sex disparities in cancer mortality and survival. Cancer Epidemiol Biomarkers Prev. 2011;20(8): 1629-1637.

52. Edgren G, Liang L, Adami HO, Chang ET. Enigmatic sex disparities in cancer incidence. Eur J Epidemiol. 2012;27(3):187-196.

53. Davoli T, Xu AW, Mengwasser KE, et al Cumulative haploinsufficiency and triplosensitivity drive aneuploidy patterns and shape the cancer genome. Cell. 2013;155 (4):948-962.

54. Minner S, Kilgue A, Stahl P, et al. Y chromosome loss is a frequent early event in urothelial bladder cancer. Pathology. 2010;42(4): 356-359.

55. Wallrapp C, Hahnel S, Boeck W, et al. Loss of the $\mathrm{Y}$ chromosome is a frequent chromosomal imbalance in pancreatic cancer and allows differentiation to chronic pancreati- tis. Int I Cancer. 2001:91(3):340-344.

56. Klatte $\mathrm{T}$, Rao PN, de Martino $M$, et al. Cytogenetic profile predicts prognosis of patients with clear cell renal cell carcinoma. J Clin Oncol. 2009;27(5):746-753.

57. Bianchi NO. Y chromosome structural and functional changes in human malignant diseases. Mutat Res 2009;682(1):21-27.

58. Germing U, Strupp C, Giagounidis A, et al Evaluation of dysplasia through detailed cytomorphology in 3156 patients from the Dusseldorf Registry on myelodysplastic syndromes. Leuk Res. 2012;36(6):727-734.

59. Maassen A, Strupp C, Giagounidis A, et al. Validation and proposals for a refinement of the WHO 2008 classification of myelodysplastic syndromes without excess of blasts. Leuk Res. 2013;37(1):64-70.

60. Duijf PH, Schultz N, Benezra R. Cancer cells preferentially lose small chromosomes. Int J Cancer. 2013;132(10):2316-2326.

61. Machiela MJ, Zhou W, Karlins E, et al Female chromosome $\mathrm{X}$ mosaicism is agerelated and preferentially affects the inactivated X chromosome. Nat Commun 2016;7:11843

62. Stone JF, Sandberg AA. Sex chromosome aneuploidy and aging. Mutat Res. 1995;338 (1-6):107-113

63. Dumanski JP, Rasi C, Lonn M, et al Mutagenesis. Smoking is associated with mosaic loss of chromosome Y. Science. 2015;347(6217):81-83.

64. Kersemaekers AM, Honecker F, Stoop H, et al. Identification of germ cells at risk for neoplastic transformation in gonadoblastoma: an immunohistochemical study for OCT3/4 and TSPY. Hum Pathol. 2005;36(5):512-521.

65. Li Y, Tabatabai ZL, Lee TL, et al. The Yencoded TSPY protein: a significant marker potentially plays a role in the pathogenesis of testicular germ cell tumors. Hum Pathol. 2007:38(10):1470-1481.

66. Vijayakumar S, Garcia D, Hensel CH, et al. The human Y chromosome suppresses the tumorigenicity of PC-3, a human prostate cancer cell line, in athymic nude mice. Genes Chromosomes Cancer. 2005;44(4): 365-372.

67. Schneider-Gadicke A, Beer-Romero P, Brown LG, Nussbaum R, Page DC. ZFX has a gene structure similar to ZFY, the putative human sex determinant, and escapes X inactivation. Cell. 1989;57(7):1247-1258.

68. Hasle $\mathrm{H}$. Turner syndrome and myelodysplastic syndrome. No reason to alert. Pediatr Hematol Oncol. 1997;19(2):179. 